\title{
ON POINTED HOPF ALGEBRAS ASSOCIATED WITH THE SYMMETRIC GROUPS
}

\author{
NICOLÁS ANDRUSKIEWITSCH, FERNANDO FANTINO \\ AND SHOUCHUAN ZHANG
}

\begin{abstract}
It is an important open problem whether the dimension of the Nichols algebra $\mathfrak{B}(\mathcal{O}, \rho)$ is finite when $\mathcal{O}$ is the class of the transpositions and $\rho$ is the sign representation, with $m \geq 6$. In the present paper, we discard most of the other conjugacy classes showing that very few pairs $(\mathcal{O}, \rho)$ might give rise to finite-dimensional Nichols algebras.
\end{abstract}

\section{Introduction and Main Result}

1.1. The context. This paper contributes to the classification of finitedimensional pointed complex Hopf algebras $H$ whose group of group-likes $G(H)$ is isomorphic to $\mathbb{S}_{m}$. Suppose we want to classify finite-dimensional pointed Hopf algebras with a fixed $G(H)=G$. As explained in [AS], the crucial step is to determine when the Nichols algebra of a Yetter-Drinfeld module over $G$ is finite-dimensional or not. Recall that irreducible YetterDrinfeld modules over $G$ are determined by a conjugacy class $\mathcal{O}$ of $G$ and an irreducible representation of the centralizer $G^{\sigma}$ of a fixed $\sigma \in \mathcal{O}$. Let $M(\mathcal{O}, \rho)$ be the corresponding Yetter-Drinfeld module and let $\mathfrak{B}(\mathcal{O}, \rho)$ denote its Nichols algebra.

1.2. Statement of the main result. Assume that $G=\mathbb{S}_{m}, 3 \leq m \in$ $\mathbb{N}$. We fix $\sigma \in \mathbb{S}_{m}$ of type $\left(1^{n_{1}}, 2^{n_{2}}, \ldots, m^{n_{m}}\right)$, which means that in the decomposition of $\sigma$ as product of disjoint cycles appear $n_{j}$ cycles of length $j$, for every $j, 1 \leq j \leq m$. We will write

$$
\sigma=A_{1} \cdots A_{m}
$$

where $A_{j}=A_{1, j} \cdots A_{n_{j}, j}$ is the product of the $n_{j}>0$ disjoint $j$-cycles $A_{1, j}$, $\ldots, A_{n_{j}, j}$ of $\sigma$. We omit $A_{j}$ when $n_{j}=0$. The even and the odd parts of $\sigma$ are

$$
\sigma_{e}:=\prod_{j \text { even }} A_{j}, \quad \sigma_{o}:=\prod_{1<j \text { odd }} A_{j}
$$

Date: October 24, 2018.

2000 Mathematics Subject Classification. 16W30; $17 \mathrm{~B} 37$.

This work was partially supported by CONICET, ANPCyT and Secyt (UNC). 
Thus, $\sigma=A_{1} \sigma_{e} \sigma_{o}$. By abuse of notation we shall say that $\sigma$ has type $\left(1^{n_{1}}, 2^{n_{2}}, \ldots, \sigma_{o}\right)$ to mean that the type of the $\sigma_{o}$ entering in $\sigma$ is arbitrary. Here is our main Theorem. See below for unexplained notation; in particular, see (5) for the meaning of $\rho_{j}$.

Theorem 1. Let $\sigma \in \mathbb{S}_{m}$ be of type $\left(1^{n_{1}}, 2^{n_{2}}, \ldots, m^{n_{m}}\right)$, let $\mathcal{O}$ be the conjugacy class of $\sigma$ and let $\rho=(\rho, V) \in \widehat{\mathbb{S}_{m}^{\sigma}}$. Assume that $\operatorname{dim} \mathfrak{B}(\mathcal{O}, \rho)<\infty$. Then $q_{\sigma \sigma}=-1$ and some of the following hold:

(i) $\left(1^{n_{1}}, 2\right), \rho_{1}=\operatorname{sgn}$ or $\epsilon, \rho_{2}=\operatorname{sgn}$.

(ii) $\left(2, \sigma_{o}\right), \sigma_{o} \neq \mathrm{id}, \rho_{2}=\operatorname{sgn}, \rho_{j}=\operatorname{Ind} \chi_{(0, \ldots, 0)} \otimes \mu_{j}$, for all $j>1$ odd.

(iii) $\left(1^{n_{1}}, 2^{3}\right), \rho_{1}=\operatorname{sgn}$ or $\epsilon, \rho_{2}=\chi_{(3)} \otimes \epsilon$ or $\chi_{(3)} \otimes \operatorname{sgn}$.

Furthermore, if $n_{1}>0$, then $\rho_{2}=\chi_{(3)} \otimes \operatorname{sgn}$.

(iv) $\left(2^{5}\right), \rho_{2}=\chi_{(5)} \otimes \epsilon$ or $\chi_{(5)} \otimes \operatorname{sgn}$.

(v) $\left(1^{n_{1}}, 4\right), \rho_{1}=\operatorname{sgn}$ or $\epsilon, \rho_{4}=\chi_{(-1)}$.

(vi) $\left(1^{n_{1}}, 4^{2}\right), \rho_{1}=\operatorname{sgn}$ or $\epsilon, \rho_{4}=\chi_{(i, i)} \otimes \operatorname{sgn}$ or $\chi_{(-i,-i)} \otimes \operatorname{sgn}$.

(vii) $(2,4), \rho=\operatorname{sgn} \otimes \epsilon$ or $\rho=\epsilon \otimes \chi_{(-1)}$.

(viii) $\left(2,4^{2}\right), \rho_{2}=\epsilon, \rho_{4}=\chi_{(i, i)} \otimes \operatorname{sgn}$ or $\chi_{(-i,-i)} \otimes \operatorname{sgn}$.

(ix) $\left(2^{2}, 4\right), \operatorname{deg} \rho_{2}=1, \rho_{4}=\chi_{(-1)}$.

We stress that in most of the cases en the previous statement, whether the dimension of the corresponding Nichols algebra is finite is an open problem; the point of the Theorem is to discard the cases not comprised in (i) to (ix).

1.3. Proof of the main result. In the previous papers AF1, AZ and in a preliminar version of the present paper, the idea was to look at abelian subracks. But we have found that the techniques based on non-abelian subracks presented in AF2 - consequences of the results in [AHS- conduct faster to a more complete analysis of Nichols algebras over symmetric groups. We use also here another technique, an extension of Gñ1, consisting in finding a suitable braided vector subspace of diagonal type not supported by an abelian subrack but "transversal" - see Proposition 3.3. We now outline the proof of the main Theorem, addressing to previous papers or to results in the present paper for proofs of the different steps.

Proof. We state the different arguments that reduce the class of possible Nichols algebras with finite dimension.

(a) $q_{\sigma \sigma}=-1$ and $\sigma$ has even order by [AZ, 2.2], cf. Lemma 2.1 below.

(b) If $j \geq 6$ is even, then $n_{j}=0$; this follows from [AF2, Ex. 2.10] for $j$ having an odd divisor, and from Proposition 3.3 for $j$ a power of 2 . Hence $\operatorname{deg} \rho_{1}=1$ by Propositions 3.8 and 3.9 , that is, $\rho_{1}=\operatorname{sgn}$ or $\epsilon$.

(c) $n_{4} \leq 2$, by [AF2, Ex. 3.10]; $n_{2} \leq 5$, by [AF2, Ex. 3.13].

(d) $\operatorname{deg} \rho_{2}=\operatorname{deg} \rho_{4}=1$, by Proposition 3.4 and [AZ, Prop. 2.6]. 
(e) Assume that there exists $j \geq 3$ such that $n_{j}>0$. Then $n_{2} \leq 2$ by AF2, Ex. 3.12]. If $n_{2}>0$, then $n_{1}=0$ by [AF2, Ex. 3.9]. Moreover, $\left(2^{2}, 4^{2}, \sigma_{o}\right)$ is excluded by Proposition 3.6.

(f) If $n_{4}>0$, then $\sigma_{o}$ is trivial by Prop. 3.7. The restrictions on the representations follow from Proposition 3.5.

(g) Assume next that for any $j \geq 3, n_{j}=0$, so that $\sigma$ is of type $\left(1^{n_{1}}, 2^{n_{2}}\right)$. Then $n_{2} \neq 2$ by [AZ, Th. 2.7] and $n_{2} \neq 4$ by [AF1, Th. 1 (B) (i)] together with [AZ, Prop. 2.6]. Also, $\rho_{2}$ should be $\chi_{\left(n_{2}\right)} \otimes \epsilon$ or $\chi_{\left(n_{2}\right)} \otimes$ sgn by [AF1, Th. 1 (B) (ii)]. Now, by Lemma 3.10 we have the restrictions on the characters in (iii) and (iv). On the other hand, the claim in (ii) for the representations $\rho_{j}, j>1$ odd, follows from Lemma 3.1 .

1.4. Comments on the cases left open. Let us say that $M(\mathcal{O}, \rho)$ has negative braiding if the Nichols algebra of any braided subspace corresponding to an abelian subrack is (twist-equivalent to) an exterior algebra.

(I) In the cases (i)-(ix) of the main Theorem, there is no family of type $\mathfrak{O}^{(2)}$ nor $\mathcal{D}_{p}^{(2)}$, for any odd prime $p$, inside the respective conjugacy classes. Besides, there is no transversal subrack of type $\mathcal{D}_{4}^{(2)}$. Moreover, it is easy to see that the corresponding braiding is negative in all cases.

(II) We know that $\operatorname{dim} \mathfrak{B}(\mathcal{O}, \rho)<\infty$ in the following cases: $\mathcal{O}$ is the class of the transpositions, $\rho=\mathrm{id} \otimes \operatorname{sgn}$ or $\epsilon \otimes \operatorname{sgn}$ (see the notation

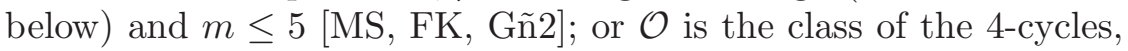
$\rho=\chi_{(-1)}$ and $m=4$ [AG, Th. 6.12]. It is an important open problem whether the dimension of $\mathfrak{B}(\mathcal{O}, \rho)$ is finite when $\mathcal{O}$ is the class of the transpositions and $m \geq 6$.

(III) The Yetter-Drinfeld modules over the group algebra $\mathbb{C} G$, with $G$ a finite group, are semisimple. The Nichols algebra of a finitedimensional reducible Yetter-Drinfeld module over $\mathbb{S}_{m}$ is always infinite-dimensional - see [HS], see also [AHS, Section 4].

\section{Preliminaries}

2.1. Generalities. We follow the conventions in AZ, AF1. We denote by $\widehat{G}$ the set of isomorphism classes of irreducible representations of a finite group $G$. We use the rack notation $x \triangleright y:=x y x^{-1}$. We set $\omega_{n}:=e^{\frac{2 \pi i}{n}}$, where $i=\sqrt{-1}$.

Let $\sigma \in G, \mathcal{O}_{\sigma}$ the conjugacy class of $\sigma$ and $\rho=(\rho, V) \in \widehat{G^{\sigma}}$. Since $\sigma \in Z\left(G^{\sigma}\right)$, the center of $G^{\sigma}$, the Schur Lemma implies that

$$
\sigma \text { acts by a scalar } q_{\sigma \sigma} \text { on } V \text {. }
$$

Lemma 2.1. [AZ, Lemma 2.2] Assume that $\sigma$ is real (i. e. $\sigma^{-1} \in \mathcal{O}_{\sigma}$ ). If $\operatorname{dim} \mathfrak{B}\left(\mathcal{O}_{\sigma}, \rho\right)<\infty$, then $q_{\sigma \sigma}=-1$ and $s$ has even order. 
The following result follows from $[\mathrm{H}]$.

Lemma 2.2. Let $W$ be a braided vector space, $U \subseteq W$ a braided vector subspace of diagonal type, $\mathcal{G}$ the generalized Dynkin diagram corresponding to $U$. If $\mathcal{G}$ contains a $t$-cycle with $t>3$, then $\operatorname{dim} \mathfrak{B}(W)=\infty$.

2.2. Symmetric groups. Let $\sigma \in \mathbb{S}_{m}$ be of type $\left(1^{n_{1}}, 2^{n_{2}}, \ldots, m^{n_{m}}\right)$. Recall the notation (11). The centralizer of $\sigma$ is isomorphic to a product $\mathbb{S}_{m}^{\sigma}=T_{1} \times \cdots \times T_{m}$, where

$$
T_{j}=\left\langle A_{1, j}, \ldots, A_{n_{j}, j}\right\rangle \rtimes\left\langle B_{1, j}, \ldots, B_{n_{j}-1, j}\right\rangle \simeq(\mathbb{Z} / j)^{n_{j}} \rtimes \mathbb{S}_{n_{j}},
$$

$1 \leq j \leq m$. We will choose $A_{1}, \ldots, A_{m}$ such that $A_{1,1}=(1), \ldots, A_{n_{1}, 1}=$ $\left(n_{1}\right), A_{1,2}=\left(n_{1}+1 n_{1}+2\right), \ldots, A_{n_{2}, 2}=\left(n_{1}+2 n_{2}-1 n_{1}+2 n_{2}\right)$, and so on. More precisely, if $1<j \leq m$ and $r:=\sum_{1 \leq k \leq j-1} k n_{k}$, then

$$
\begin{aligned}
& A_{l, j}:=\left(\begin{array}{llll}
r+(l-1) j+1 & r+(l-1) j+2 & \cdots & r+l j
\end{array}\right), \\
& B_{h, j}:=(r+(h-1) j+1 \quad r+h j+1)(r+(h-1) j+2 \quad r+h j+2) \\
& \cdots(r+h j \quad r+(h+1) j)
\end{aligned}
$$

for all $l, h$, with $1 \leq l \leq n_{j}, 1 \leq h \leq n_{j}-1$. Notice that $B_{h, j}$ is an involution.

Let $\rho=(\rho, V) \in \widehat{\mathbb{S}_{m}^{\sigma}} ;$ so

$$
\rho=\rho_{1} \otimes \cdots \otimes \rho_{m},
$$

where $\rho_{j} \in \widehat{T_{j}}$ has the form

$$
\left.\rho_{j}=\operatorname{Ind}_{\mathbb{Z}_{j}^{n_{j}} \rtimes \mathbb{S}_{n_{j}}^{\chi_{j}}} \frac{\mathbb{R}_{j}^{n_{j}} \rtimes \mathbb{S}_{n_{j}}}{\chi_{j}} \otimes \mu_{j}\right)
$$

with $\chi_{j} \in \widehat{\mathbb{Z}_{j}^{n_{j}}}$ and $\mu_{j} \in \widehat{\mathbb{S}_{n_{j}}^{\chi_{j}}}$ - see [S, Section 8.2]. Here $\mathbb{S}_{n_{j}}^{\chi_{j}}$ denotes the isotropy subgroup of $\chi_{j}$ under the induced action of $\mathbb{S}_{n_{j}}$ over $\widehat{\mathbb{Z}_{j}^{n_{j}}}$. Actually, $\chi_{j}$ is of the form $\chi_{\left(t_{1, j}, \ldots, t_{n_{j}, j}\right)}$, where $0 \leq t_{1, j}, \ldots, t_{n_{j}, j} \leq j-1$ are such that

$$
\chi_{\left(t_{1, j}, \ldots, t_{n_{j}, j}\right)}\left(A_{l, j}\right)=\omega_{j}^{t_{l, j}}, \quad 1 \leq l \leq n_{j} .
$$

Notice that if $\rho_{j}$ is as in (5), then

$$
\operatorname{deg} \rho_{j}=\left[\mathbb{S}_{n_{j}}: \mathbb{S}_{n_{j}}^{\left(\chi_{j}\right)}\right] \operatorname{deg} \mu_{j}
$$

Remark 2.3. Since every $A_{j}$ belongs to $Z\left(\mathbb{S}_{m}^{\sigma}\right), A_{j}$ acts by a scalar $q_{A_{j}}$ on $V$. Thus,

$$
q_{\sigma \sigma}=q_{e} q_{o}, \quad \text { where } q_{e}=\prod_{j \text { even }} q_{A_{j}} \text { and } q_{o}=\prod_{1<j \text { odd }} q_{A_{j}} .
$$


Remark 2.4. Assume that $\operatorname{deg}(\rho)=1$; that is, $\operatorname{deg}\left(\rho_{j}\right)=1$, for all $j$. Then

$$
\mathbb{S}_{n_{j}}^{\left(\chi_{j}\right)}=\mathbb{S}_{n_{j}} \quad \text { and } \quad \mu_{j}=\epsilon \text { or } \operatorname{sgn} \in \widehat{\mathbb{S}_{n_{j}}}, \quad \text { for all } j,
$$

by (7). Hence, we have that $t_{j}:=t_{1, j}=\cdots=t_{n_{j}, j}$, for every $j$, and $\rho_{j}=\chi_{j} \otimes \mu_{j}$. In that case, we will denote $\chi_{j}=\chi_{\left(t_{j}, \ldots, t_{j}\right)}$ by $\overrightarrow{\chi_{t_{j}}}$. Thus, for every $j$ there exists $t_{j}$, with $0 \leq t_{j} \leq j-1$, such that

$$
\rho=\left(\overrightarrow{\chi_{t_{1}}} \otimes \mu_{1}\right) \otimes \cdots \otimes\left(\overrightarrow{\chi_{t_{m}}} \otimes \mu_{m}\right) .
$$

If $n_{j}=0$ or 1 , then $\rho_{j}$ is just the trivial representation. We will denote

$$
\mathbf{t}:=\left(t_{1}, \ldots, t_{m}\right),
$$

which is a $m$-tuple that depends on $\rho$. Any one-dimensional representation of $\mathbb{S}_{m}^{\sigma}$ is completely determined by $\left(\mu_{1}, \ldots, \mu_{m}\right)$ and $\mathbf{t}$ as above.

\section{NEW RESTRICTIONS ON ORBITS AND CHARACTERS}

Our first new result restricts the possibilities for the exponents $t_{-,-}$'s of the representation $\rho$. The proof is an application of the technique of abelian subracks.

Lemma 3.1. Let $\rho=(\rho, V) \in \widehat{\mathbb{S}_{m}^{\sigma}}$. If there exist $j, l$, with $1 \leq j \leq m$ and $1 \leq l \leq n_{j}$, such that $\omega_{j}^{4 t_{l, j}} \neq 1$, then $\operatorname{dim} \mathfrak{B}\left(\mathcal{O}_{\sigma}, \rho\right)=\infty$.

Proof. Notice that $j \neq 1,2$. Let $N=\sum_{j \geq 3} n_{j}$. We consider two cases.

(a) Assume that $N=1$. In this case, the type of $\sigma$ is $\left(1^{n_{1}}, 2^{n_{2}}, j\right)$. Then $\rho_{j}=\chi_{t_{j}}$, for some $t_{j}, 0<t_{j} \leq j-1$, and $q_{\sigma \sigma}= \pm \omega_{j}^{t_{j}} \neq \pm 1$, by hypothesis. Now the result follows from Lemma 2.1.

(b) Assume that $N>1$. By Lemma 2.1, we may suppose that $q_{\sigma \sigma}=-1$. There exists $v \in V-0$ such that $\rho\left(A_{l, j}\right) v=\omega_{j}^{t_{l, j}} v$. We define $\sigma_{1}:=\sigma$, $\sigma_{2}:=\sigma A_{l, j}^{-2}, \sigma_{3}:=\sigma_{2}^{-1}$ and $\sigma_{4}:=\sigma^{-1}$; clearly, these are four different elements. Let $\tau=\left(\begin{array}{ll}i_{1} & i_{2} \\ \cdots & i_{j}\end{array}\right)$ be a $j$-cycle. We define

$$
g_{\tau}:= \begin{cases}\left(i_{2} i_{j}\right)\left(i_{3} i_{j-1}\right) \cdots\left(i_{l} i_{l+2}\right) & , \text { if } j=2 l \text { is even, } \\ \left(i_{2} i_{j}\right)\left(i_{3} i_{j-1}\right) \cdots\left(i_{l+1} i_{l+2}\right) & , \text { if } j=2 l+1 \text { is odd. }\end{cases}
$$

Thus, $g_{\tau}$ is an involution such that $\tau^{-1}=g_{\tau} \tau g_{\tau}$.

We choose $g_{1}:=\mathrm{id}, g_{2}:=g_{A_{l, j}}$, see (11), $g_{4}:=g_{2} g_{3}$ and

$$
g_{3}:=\prod_{\substack{k \neq j \\ 1 \leq h \leq n_{k}}} g_{A_{h, k}} \cdot \prod_{\substack{1 \leq h \leq n_{j} \\ h \neq l}} g_{A_{h, j}} .
$$


Then $\sigma_{r}=g_{r} \sigma g_{r}^{-1}, r=1,2,3,4$, and we have the following relations

$$
\begin{array}{llll}
\sigma_{1} g_{1}=g_{1} \sigma_{1}, & \sigma_{1} g_{2}=g_{2} \sigma_{2}, & \sigma_{1} g_{3}=g_{3} \sigma_{3}, & \sigma_{1} g_{4}=g_{4} \sigma_{4}, \\
\sigma_{2} g_{1}=g_{1} \sigma_{2}, & \sigma_{2} g_{2}=g_{2} \sigma_{1}, & \sigma_{2} g_{3}=g_{3} \sigma_{4}, & \sigma_{2} g_{4}=g_{4} \sigma_{3}, \\
\sigma_{3} g_{1}=g_{1} \sigma_{3}, & \sigma_{3} g_{2}=g_{2} \sigma_{4}, & \sigma_{3} g_{3}=g_{3} \sigma_{1}, & \sigma_{3} g_{4}=g_{4} \sigma_{2}, \\
\sigma_{4} g_{1}=g_{1} \sigma_{4}, & \sigma_{4} g_{2}=g_{2} \sigma_{3}, & \sigma_{4} g_{3}=g_{3} \sigma_{2}, & \sigma_{4} g_{4}=g_{4} \sigma_{1} .
\end{array}
$$

It is not difficult to see that $W:=\mathbb{C}$-span $\left\{g_{1} v, g_{2} v, g_{3} v, g_{4} v\right\}$ is a braided vector subspace of diagonal type of $M\left(\mathcal{O}_{\sigma}, \rho\right)$, with braiding matrix

$$
\mathcal{Q}=\left(\begin{array}{cccc}
-1 & \omega_{j}^{2 t_{l, j}} & \omega_{j}^{-2 t_{l, j}} & -1 \\
\omega_{j}^{2 t_{l, j}} & -1 & -1 & \omega_{j}^{-2 t_{l, j}} \\
\omega_{j}^{-2 t_{l, j}} & -1 & -1 & \omega_{j}^{2 t_{l, j}} \\
-1 & \omega_{j}^{-2 t_{l, j}} & \omega_{j}^{2 t_{l, j}} & -1
\end{array}\right)
$$

Since $\omega_{j}^{4 t_{l, j}} \neq 1$ the generalized Dynkin diagram is of the form given by Figure 1, Therefore, $\operatorname{dim} \mathfrak{B}\left(\mathcal{O}_{\sigma}, \rho\right)=\infty$, by Lemma 2.2.

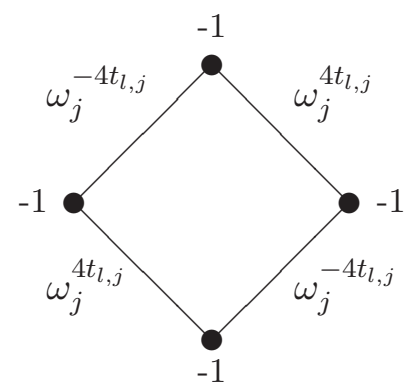

FigURE 1.

Remark 3.2. The previous Lemma implies that if $\operatorname{dim} \mathfrak{B}\left(\mathcal{O}_{\sigma}, \rho\right)<\infty$, with $\rho \in \widehat{\mathbb{S}_{m}^{\sigma}}$, then the scalars $q_{e}$ and $q_{o}$ given in Remark 2.3 must be $q_{o}=1$ and $q_{e}=-1$; moreover, $t_{-, j}=0$, for all $j$ odd.

Our next result discards the appearance of cycles of length $j>4$, where $j$ is a power of 2 . The proof is an application of the technique of transversal abelian subspaces. We also need Lemma 3.1.

Proposition 3.3. Let $\sigma \in \mathbb{S}_{m}$, O the conjugacy class of $\sigma$ and $\rho \in \widehat{\mathbb{S}_{m}^{\sigma}}$. If the type of $\sigma$ is $\left(1^{n_{1}}, 2^{n_{2}}, 4^{n_{4}}, 8^{n_{8}}, \ldots,\left(2^{k}\right)^{n_{2^{k}}}, \sigma_{o}\right)$, with $k \geq 3$ and $n_{2^{k}} \geq 1$, then $\operatorname{dim} \mathfrak{B}(\mathcal{O}, \rho)=\infty$.

Proof. We may assume that $\sigma$ is of type $\left(2^{n_{2}}, 4^{n_{4}}, 8^{n_{8}}, \ldots,\left(2^{k}\right)^{n_{2} k}\right)$, by [AZ, Prop. 2.6]. If $q_{\sigma \sigma} \neq-1$ or if $n_{2^{k}} \geq 3$, then the result follows from Lemma 
2.1 and [AF2, Ex. 3.10], respectively. Assume that $q_{\sigma \sigma}=-1$ and $n_{2^{k}} \leq 2$. We consider two cases.

(I) Assume that $n_{2^{k}}=1$. Let $\alpha=\left(i_{1} i_{2} \cdots i_{2^{k}}\right)$ be the $2^{k}$-cycle appearing in the decomposition of $\sigma$ as product of disjoint cycles, and we call

$$
\mathbf{I}:=\left(i_{1} i_{3} i_{5} \cdots i_{2^{k}-1}\right) \quad \text { and } \quad \mathbf{P}:=\left(i_{2} i_{4} i_{6} \cdots i_{2^{k}}\right) .
$$

In the proof of [AF2, Lemma 2.11], it was shown that

(a) $\mathbf{I}$ and $\mathbf{P}$ are disjoint $2^{k-1}$-cycles,

(b) $\alpha^{2}=\mathbf{I P}$

(c) $\alpha \mathbf{I} \alpha^{-1}=\mathbf{P}$, (hence $\sigma \mathbf{I} \sigma^{-1}=\mathbf{P}$ ),

(d) $\mathbf{P}^{t} \alpha \mathbf{P}^{t}=\alpha^{2 t+1}$, for all integer $t$.

For every $l \in \mathbb{Z}_{4}$, we call $\alpha_{l}=\mathbf{P}^{2^{k-3} l} \alpha \mathbf{P}^{-2^{k-3} l}$ and we define as in AF2, $(2.17)]$

$$
\sigma_{l}:=\mathbf{P}^{2^{k-3} l} \sigma \mathbf{P}^{-2^{k-3} l}
$$

Then $\left(\sigma_{l}\right)_{l \in \mathbb{Z}_{4}}$ is of type $\mathcal{D}_{4}$ in the sense of [AF2, Def. 2.2].

Claim 1. (i) $\alpha_{2}=\alpha^{2^{k-1}+1}$, (ii) $\alpha_{3}=\alpha_{1}^{2^{k-1}+1}$.

Proof. Notice that $\mathbf{P}^{2^{k-2}}$ is an involution, since $\mathbf{P}$ is a $2^{k-1}$-cycle. Then $\alpha_{2}=\mathbf{P}^{2^{k-3} 2} \alpha \mathbf{P}^{-2^{k-3} 2}=\mathbf{P}^{2^{k-2}} \alpha \mathbf{P}^{2^{k-2}}=\alpha^{22^{k-2}+1}=\alpha^{2^{k-1}+1}$, by (d) above. Analogously,

$$
\begin{aligned}
\alpha_{3} & =\mathbf{P}^{2^{k-3} 3} \alpha \mathbf{P}^{-2^{k-3} 3}=\mathbf{P}^{2^{k-3}} \mathbf{P}^{2^{k-2}} \alpha \mathbf{P}^{-2^{k-2}} \mathbf{P}^{-2^{k-3}} \\
& =\mathbf{P}^{2^{k-3}} \alpha^{2^{k-1}+1} \mathbf{P}^{-2^{k-3}}=\left(\mathbf{P}^{2^{k-3}} \alpha \mathbf{P}^{-2^{k-3}}\right)^{2^{k-1}+1}=\alpha_{1}^{2^{k-1}+1}
\end{aligned}
$$

as desired.

Notice that (i) implies that $\sigma_{2}=\sigma^{2^{k-1}+1}$ because $\sigma^{2^{k-1}}=\alpha^{2^{k-1}}$. Analogously, $\sigma_{3}=\sigma_{1}^{2^{k-1}+1}$. If we define $\tau_{l}:=\sigma_{l}^{-1}$, for all $l$, then $\left(\sigma_{l}\right)_{l \in \mathbb{Z}_{4}} \cup\left(\tau_{l}\right)_{l \in \mathbb{Z}_{4}}$ is of type $\mathcal{D}_{4}^{(2)}$. We define $g:=\left(\begin{array}{lll}i_{1} & i_{2^{k}}\end{array}\right)\left(\begin{array}{ll}i_{2} & i_{2^{k}-1}\end{array}\right) \cdots\left(\begin{array}{ll}i_{2^{k-1}-1} & i_{2^{k-1}+1}\end{array}\right)$. Then $g$ is an involution in $\mathbb{S}_{m}$ such that $g \triangleright \sigma=\sigma^{-1}$. We define $g_{l}:=\mathbf{P}^{2^{k-3} l}$ and

$$
h_{l}:=g_{l} g, \quad l \in \mathbb{Z}_{4} .
$$

Clearly, $h_{l} \triangleright \sigma=\tau_{l}, l \in \mathbb{Z}_{4}$.

Claim 2. We set $r:=2^{k-3}$. We have the following multiplication tables: 


\begin{tabular}{c|cccc}
$\cdot$ & $g_{0}$ & $g_{1}$ & $g_{2}$ & $g_{3}$ \\
\hline$\sigma_{0}$ & $g_{0} \sigma$ & $g_{3} \sigma \alpha^{2 r}$ & $g_{2} \sigma_{2}$ & $g_{1} \sigma \alpha^{-2 r}$ \\
$\sigma_{1}$ & $g_{2} \sigma \alpha^{-2 r}$ & $g_{1} \sigma$ & $g_{0} \sigma \alpha^{2 r}$ & $g_{3} \sigma_{2}$ \\
$\sigma_{2}$ & $g_{0} \sigma_{2}$ & $g_{3} \sigma \alpha^{-2 r}$ & $g_{2} \sigma$ & $g_{1} \sigma \alpha^{2 r}$ \\
$\sigma_{3}$ & $g_{2} \sigma \alpha^{2 r}$ & $g_{1} \sigma_{2}$ & $g_{0} \sigma \alpha^{-2 r}$ & $g_{3} \sigma$ \\
$\tau_{0}$ & $g_{0} \sigma^{-1}$ & $g_{3} \sigma^{-1} \alpha^{2 r}$ & $g_{2} \sigma_{2}^{-1}$ & $g_{1} \sigma^{-1} \alpha^{-2 r}$ \\
$\tau_{1}$ & $g_{2} \sigma^{-1} \alpha^{-2 r}$ & $g_{1} \sigma^{-1}$ & $g_{0} \sigma^{-1} \alpha^{2 r}$ & $g_{3} \sigma_{2}^{-1}$ \\
$\tau_{2}$ & $g_{0} \sigma_{2}^{-1}$ & $g_{3} \sigma^{-1} \alpha^{-2 r}$ & $g_{2} \sigma^{-1}$ & $g_{1} \sigma^{-1} \alpha^{2 r}$ \\
$\tau_{3}$ & $g_{2} \sigma^{-1} \alpha^{2 r}$ & $g_{1} \sigma_{2}^{-1}$ & $g_{0} \sigma^{-1} \alpha^{-2 r}$ & $g_{3} \sigma^{-1}$
\end{tabular}

\begin{tabular}{c|cccc}
$\cdot$ & $h_{0}$ & $h_{1}$ & $h_{2}$ & $h_{3}$ \\
\hline$\sigma_{0}$ & $h_{0} \sigma^{-1}$ & $h_{3} \sigma^{-1} \alpha^{-2 r}$ & $h_{2} \sigma_{2}^{-1}$ & $h_{1} \sigma^{-1} \alpha^{2 r}$ \\
$\sigma_{1}$ & $h_{2} \sigma^{-1} \alpha^{2 r}$ & $h_{1} \sigma^{-1}$ & $h_{0} \sigma^{-1} \alpha^{-2 r}$ & $h_{3} \sigma_{2}^{-1}$ \\
$\sigma_{2}$ & $h_{0} \sigma_{2}^{-1}$ & $h_{3} \sigma^{-1} \alpha^{2 r}$ & $h_{2} \sigma^{-1}$ & $h_{1} \sigma^{-1} \alpha^{-2 r}$ \\
$\sigma_{3}$ & $h_{2} \sigma^{-1} \alpha^{-2 r}$ & $h_{1} \sigma_{2}^{-1}$ & $h_{0} \sigma^{-1} \alpha^{2 r}$ & $h_{3} \sigma^{-1}$ \\
$\tau_{0}$ & $h_{0} \sigma$ & $h_{3} \sigma \alpha^{-2 r}$ & $h_{2} \sigma_{2}$ & $h_{1} \sigma \alpha^{2 r}$ \\
$\tau_{1}$ & $h_{2} \sigma \alpha^{2 r}$ & $h_{1} \sigma$ & $h_{0} \sigma \alpha^{-2 r}$ & $h_{3} \sigma_{2}$ \\
$\tau_{2}$ & $h_{0} \sigma_{2}$ & $h_{3} \sigma \alpha^{2 r}$ & $h_{2} \sigma$ & $h_{1} \sigma \alpha^{-2 r}$ \\
$\tau_{3}$ & $h_{2} \sigma \alpha^{-2 r}$ & $h_{1} \sigma_{2}$ & $h_{0} \sigma \alpha^{2 r}$ & $h_{3} \sigma$
\end{tabular}

Proof. The multiplications $\sigma_{i} g_{j}$ follow by straightforward computations, using the fact that $\alpha^{2^{k-1}}=\sigma^{2^{k-1}}$. For the multiplications $\sigma_{i} h_{j}$, use that $h_{i \triangleright j}^{-1} \sigma_{i} h_{j}=\left(g_{i \triangleright j}^{-1} \sigma_{i} g_{j}\right)^{-1}$, and the result follows. The rest can be checked in an analogous way. Notice that $g_{i \triangleright j}^{-1} \tau_{i} g_{j}=\left(h_{i \triangleright j}^{-1} \tau_{i} h_{j}\right)^{-1}$.

Our assumption $q_{\sigma \sigma}=-1$ implies that $\rho\left(\sigma_{2}\right)=-\mathrm{Id}$. On the other hand, we have that $\rho(\alpha)=\omega_{2^{k}}^{t_{2 k}}$, and, by Lemma 3.1, we can suppose that

$$
t_{2^{k}}=0, \quad 2^{k-2}, \quad 2^{k-1} \quad \text { or } 3 \cdot 2^{k-2} .
$$

Hence, $\rho\left(\sigma \alpha^{2 r}\right)=-\omega_{2^{k}}^{2 r t} 2^{k} \mathrm{Id}=-i^{t} 2^{k} \mathrm{Id}= \pm \mathrm{Id}$, with $i=\sqrt{-1}$, because of (13). Also, $\rho\left(\sigma \alpha^{-2 r}\right)=-\omega_{2^{k}}^{-2 r t_{2^{k}}} \mathrm{Id}=-i^{-t_{2^{k}}} \mathrm{Id}= \pm \mathrm{Id}$. Moreover,

$$
\rho\left(\sigma \alpha^{2 r}\right)=\rho\left(\sigma \alpha^{-2 r}\right)=-\lambda \mathrm{Id},
$$

with $\lambda:=i^{t_{2 k}}$. Thus, $\rho\left(\sigma^{-1} \alpha^{2 r}\right)=\rho\left(\sigma^{-1} \alpha^{-2 r}\right)=-\lambda$ Id.

Let $v, w \in V-0$. We define $W:=\mathbb{C}$-span of $\left\{u_{l}, v_{l} \mid l \in \mathbb{Z}_{4}\right\}$, where

$$
\begin{array}{ll}
u_{1}:=g_{0} v+g_{2} v, & w_{1}:=h_{0} w+h_{2} w, \\
u_{2}:=g_{0} v-g_{2} v, & w_{2}:=h_{0} w-h_{2} w, \\
u_{3}:=g_{1} v+g_{3} v, & w_{3}:=h_{1} w+h_{3} w, \\
u_{4}:=g_{1} v-g_{3} v, & w_{4}:=h_{1} w-h_{3} w .
\end{array}
$$


By straightforward computations, we can see that $W$ is a braided vector subspace of $M\left(\mathcal{O}_{\sigma}, \rho\right)$ of Cartan type with matrix of coefficients given by

$$
\left(\begin{array}{ll}
Q & Q \\
Q & Q
\end{array}\right), \text { where } Q=\left(\begin{array}{cccc}
-1 & -1 & -\lambda & \lambda \\
-1 & -1 & -\lambda & \lambda \\
-\lambda & \lambda & -1 & -1 \\
-\lambda & \lambda & -1 & -1
\end{array}\right)
$$

and Dynkin diagram given by

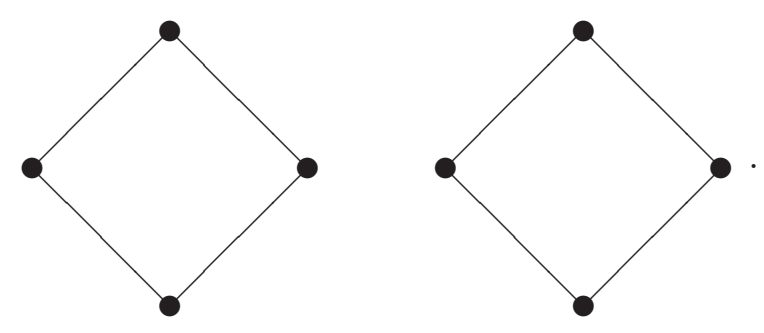

FiguRE 2.

which is not of finite type. Therefore, $\operatorname{dim} \mathfrak{B}\left(\mathcal{O}_{\sigma}, \rho\right)=\infty$.

(II) Assume that $n_{2^{k}}=2$. Let $A_{1,2^{k}}=\left(i_{1} i_{2} \cdots i_{2^{k}}\right)$ and $A_{2,2^{k}}=$ $\left(i_{2^{k}+1} i_{2^{k}+2} \cdots i_{2^{k+1}}\right)$ the two $2^{k}$-cycles appearing in $\sigma$, and let $\mathbf{I}=\mathbf{I}_{1} \mathbf{I}_{2}$ and $\mathbf{P}=\mathbf{P}_{1} \mathbf{P}_{2}$, with

$$
\begin{array}{rlrl}
\mathbf{I}_{1} & :=\left(i_{1} i_{3} \cdots i_{2^{k}-1}\right), & \mathbf{I}_{2}:=\left(i_{2^{k}+1} i_{2^{k}+3} \cdots i_{2^{k+1}-1}\right), \\
\mathbf{P}_{1}:=\left(i_{2} i_{4} \cdots i_{2^{k}}\right), & \mathbf{P}_{2}:=\left(i_{2^{k}+2} i_{2^{k}+4} \cdots i_{2^{k+1}}\right) .
\end{array}
$$

Now, we take $\sigma_{l}$ as in (12) and we proceed in an analogous way as in (I).

We next examine the possible $\rho$ 's when the type of $\sigma$ is

$$
\left(2^{n_{2}}, 4^{n_{4}}\right), \quad \text { with } n_{2} \leq 5 \text { and } n_{4} \leq 2 .
$$

Our first result for this question follows by performing the argument given in [AF1, Th. 4].

Proposition 3.4. Let $\sigma \in \mathbb{S}_{m}, \mathcal{O}$ the conjugacy class of $\sigma$ of type $\left(2^{n_{2}}, 4^{n_{4}}\right)$ and $\rho \in \widehat{\mathbb{S}_{m}^{\sigma}}$. If $\operatorname{deg} \rho>1$, then $\operatorname{dim} \mathfrak{B}(\mathcal{O}, \rho)=\infty$.

The next proposition is proved by the technique of non-abelian subracks.

Proposition 3.5. Let $\sigma \in \mathbb{S}_{m}$ of type $\left(2^{n_{2}}, 4^{2}\right)$, $\mathcal{O}$ the conjugacy class of $\sigma$ and $\rho \in \widehat{\mathbb{S}_{m}^{\sigma}}$, with $\operatorname{deg}(\rho)=1$. If $\operatorname{dim} \mathfrak{B}(\mathcal{O}, \rho)<\infty$, then $\rho_{4}=\chi_{(i, i)} \otimes \operatorname{sgn}$ or $\chi_{(-i,-i)} \otimes \operatorname{sgn}$, where $i=\sqrt{-1}$. 
Proof. We assume that $q_{\sigma \sigma}=-1$, by Lemma 2.1. Since $\operatorname{deg}(\rho)=1$, $\operatorname{deg}\left(\rho_{2}\right)=\operatorname{deg}\left(\rho_{4}\right)=1$. Let $A_{1,4}=\left(\begin{array}{llll}j_{1} & j_{2} & j_{3} & j_{4}\end{array}\right)$ and $A_{2,4}=\left(\begin{array}{llll}j_{5} & j_{6} & j_{7} & j_{8}\end{array}\right)$ the two 4-cycles that appear in the decomposition of $\sigma$ as product of disjoint cycles. We write $A_{4}=A_{1,4} A_{2,4}$. Since $\operatorname{deg} \rho=1$ we have that $\rho\left(A_{1,4}\right)=\rho\left(A_{2,4}\right)=\omega_{4}^{t_{4}}$, with $0 \leq t_{4} \leq 3-$ see Remark 2.4. Then $\rho_{4}=\chi_{\left(\omega_{4}^{t_{4}}, \omega_{4}^{t_{4}}\right)} \otimes \mu_{4}$, with $\mu_{4}=\epsilon$ ó sgn, i. e. the trivial or sign representation of $\mathbb{Z}_{2}$. Then $\rho\left(A_{4}\right)= \pm 1$. We consider two cases.

$\operatorname{CASE}(I): q_{A_{4}}=1$. Then $\rho\left(A_{1,4}\right)=\rho\left(A_{2,4}\right)=1$ ó -1 . We define $s_{1}:=A_{4}, s_{6}:=A_{1,4} A_{2,4}^{-1}, t_{1}:=A_{1,4}^{-1} A_{2,4}, t_{6}:=s_{1}^{-1}$,

$$
\begin{array}{lll}
s_{2}:=A_{1,4}\left(\begin{array}{llll}
j_{5} & j_{6} & j_{8} & j_{7}
\end{array}\right), & s_{3}:=A_{1,4}\left(\begin{array}{llll}
j_{5} & j_{7} & j_{6} & j_{8}
\end{array}\right), \\
s_{4}:=A_{1,4}\left(\begin{array}{llllll}
j_{5} & j_{7} & j_{8} & j_{6}
\end{array}\right), & s_{5}:=A_{1,4}\left(\begin{array}{lllll}
j_{5} & j_{8} & j_{6} & j_{7}
\end{array}\right), \\
t_{2}:=\left(\begin{array}{llllll}
j_{1} & j_{4} & j_{3} & j_{2}
\end{array}\right)\left(\begin{array}{llllll}
j_{5} & j_{6} & j_{8} & j_{7}
\end{array}\right), & t_{3}:=\left(\begin{array}{llllll}
j_{1} & j_{4} & j_{3} & j_{2}
\end{array}\right)\left(\begin{array}{lllll}
j_{5} & j_{7} & j_{6} & j_{8}
\end{array}\right), \\
t_{4}:=\left(\begin{array}{llllll}
j_{1} & j_{4} & j_{3} & j_{2}
\end{array}\right)\left(\begin{array}{lllll}
j_{5} & j_{7} & j_{8} & j_{6}
\end{array}\right), & t_{5}:=\left(\begin{array}{lllll}
j_{1} & j_{4} & j_{3} & j_{2}
\end{array}\right)\left(\begin{array}{llll}
j_{5} & j_{8} & j_{6} & j_{7}
\end{array}\right) .
\end{array}
$$

Let $\alpha=\sigma s_{1}^{-1}$ and we define $\sigma_{j}:=s_{j} \alpha, \tau_{j}=t_{j} \alpha, 1 \leq j \leq 6$. It is easy to see that $(\sigma, \tau)$ is of type $\mathfrak{O}^{(2)}-$ see [AF2, Section 4]. Now, $\tau_{1}=A_{1,4}^{-1} A_{2,4} \alpha=$ $\sigma A_{1,4}^{-2}, \sigma_{6}=A_{1,4} A_{2,4}^{-1} \alpha=\sigma A_{2,4}^{-2}$. We choose $g:=(12)(34)$; then $g \triangleright \sigma_{1}=\tau_{1}$, $g^{-1} \sigma_{1} g=\tau_{1}=\sigma A_{1,4}^{-2}$ y $g^{-1} \sigma_{6} g=g^{-1} A_{1,4} A_{2,4}^{-1} g \alpha=A_{1,4}^{-1} A_{2,4}^{-1} \alpha=\sigma s_{1}^{-2}$. This implies that $\rho\left(\tau_{1}\right)=\rho\left(g^{-1} \sigma_{1} g\right)=-\rho\left(A_{1,4}^{-2}\right)=-1, \rho\left(\sigma_{6}\right)=-\rho\left(A_{2,4}^{-2}\right)=-1$ y $\rho\left(g^{-1} \sigma_{6} g\right)=-\rho\left(s_{1}^{-2}\right)=-1$. Thus, we are in the situation of [AF2, Th. 4.11], and $\operatorname{dim} \mathfrak{B}(\mathcal{O}, \rho)=\infty$.

CASE (II): $q_{A_{4}}=-1$. Then $\rho\left(A_{1,4}\right)=\rho\left(A_{2,4}\right)=i$ ó $-i$, where $i=\sqrt{-1}$. Let $\mu_{4}=\epsilon$. We define $s_{1}:=A_{4}$,

$s_{2}:=\left(\begin{array}{llll}j_{1} & j_{2} & j_{4} & j_{3}\end{array}\right)\left(\begin{array}{llll}j_{5} & j_{6} & j_{8} & j_{7}\end{array}\right), \quad s_{3}:=\left(\begin{array}{llllll}j_{1} & j_{3} & j_{2} & j_{4}\end{array}\right)\left(\begin{array}{llll}j_{5} & j_{7} & j_{6} & j_{8}\end{array}\right)$, $s_{4}:=s_{2}^{-1}, s_{5}:=s_{3}^{-1}, s_{6}:=s_{1}^{-1}, t_{1}:=\left(\begin{array}{lllll}j_{1} & j_{6} & j_{3} & j_{8}\end{array}\right)\left(\begin{array}{llll}j_{2} & j_{7} & j_{4} & j_{5}\end{array}\right)$, $t_{2}:=\left(\begin{array}{llll}j_{1} & j_{6} & j_{4} & j_{7}\end{array}\right)\left(\begin{array}{llll}j_{2} & j_{8} & j_{3} & j_{5}\end{array}\right), \quad t_{3}:=\left(\begin{array}{lllll}j_{1} & j_{7} & j_{2} & j_{8}\end{array}\right)\left(\begin{array}{llll}j_{3} & j_{6} & j_{4} & j_{5}\end{array}\right)$, $t_{4}:=t_{2}^{-1}, t_{5}:=t_{3}^{-1}$ y $t_{6}:=t_{1}^{-1}$. Notice that $t_{1}=A_{1,4} A_{2,4} B_{1,4}$, where $B:=\left(\begin{array}{ll}j_{1} & j_{5}\end{array}\right)\left(\begin{array}{ll}j_{2} & j_{6}\end{array}\right)\left(j_{3} j_{7}\right)\left(j_{4} j_{8}\right)$.

Let $\alpha=\sigma s_{1}^{-1}$ and we define $\sigma_{j}:=s_{j} \alpha, \tau_{j}=t_{j} \alpha, 1 \leq j \leq 6$. It is easy to see that $(\sigma, \tau)$ is of type $\mathfrak{O}^{(2)}$. Now, $\tau_{1}=A_{1,4} A_{2,4} B_{1,4} \alpha=\sigma B_{1,4}$, $\sigma_{6}=\sigma A_{4}^{-2}$. We choose $g:=(26)(48)$; then $g \triangleright \sigma_{1}=\tau_{1}, g^{-1} \sigma_{1} g=\tau_{1}$ and $g^{-1} \sigma_{6} g=g^{-1} A_{1,4}^{-1} A_{2,4}^{-1} g \alpha=\sigma A_{4}^{-2} B_{1,4}$. Then $\rho\left(\tau_{1}\right)=\rho\left(g^{-1} \sigma_{1} g\right)=\rho\left(\sigma_{6}\right)=$ $\rho\left(g^{-1} \sigma_{6} g\right)=-1$, and $\operatorname{dim} \mathfrak{B}(\mathcal{O}, \rho)=\infty$, again by [AF2, Th. 4.11].

Our next task is to discard the type $\left(2^{2}, 4^{2}, \sigma_{o}\right)$; we do this by the technique of transversal diagonal braided subspaces. 
Proposition 3.6. Let $\sigma \in \mathbb{S}_{m}$ of type $\left(2^{2}, 4^{2}, \sigma_{o}\right)$, $\mathcal{O}$ the conjugacy class of $\sigma$ and $\rho \in \widehat{\mathbb{S}_{m}^{\sigma}}$, with $\operatorname{deg}(\rho)=1$. Then $\operatorname{dim} \mathfrak{B}(\mathcal{O}, \rho)=\infty$.

Proof. By [AZ, Prop. 2.6], we may assume that $\sigma \in \mathbb{S}_{12}$ is of type $\left(2^{2}, 4^{2}\right)$.

If $\rho_{4} \neq \chi_{(i, i)} \otimes \operatorname{sgn}, \chi_{(-i,-i)} \otimes \operatorname{sgn}$, where $i=\sqrt{-1}$, then the result follows by Proposition 3.5. Assume that $\rho_{4}=\chi_{(i, i)} \otimes \operatorname{sgn}$ or $\chi_{(-i,-i)} \otimes$ sgn, with $i=\sqrt{-1}$. Following the notation given in the preliminaries, we take $A_{1,2}=$

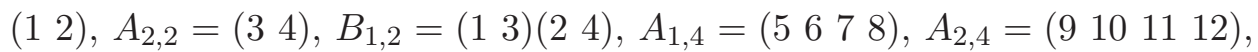
$B_{1,4}=\left(\begin{array}{ll}5 & 9\end{array}\right)\left(\begin{array}{ll}6 & 10\end{array}\right)\left(\begin{array}{l}7 \\ 11\end{array}\right)(812)$. We call $A_{2}=A_{1,2} A_{2,2}, A_{4}=A_{1,4} A_{2,4}$ and

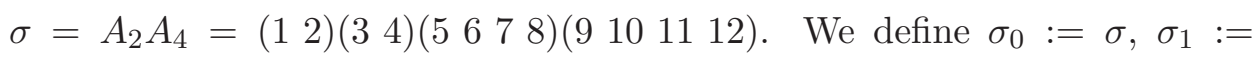
(1 2)(3 4)(5 97 11)(6 128 10), $\sigma_{2}:=\sigma_{0}^{-1}, \sigma_{3}:=\sigma_{1}^{-1}$, $\tau_{0}:=\left(\begin{array}{ll}1 & 3\end{array}\right)(24)\left(\begin{array}{lll}5 & 6 & 8\end{array}\right)\left(91011\right.$ 12), $\tau_{1}:=\left(\begin{array}{lll}1 & 3\end{array}\right)(2$ 4)(5 97 11)(6 128 10), $\tau_{2}:=\tau_{0}^{-1}$ and $\tau_{3}:=\tau_{1}^{-1}$. It is easy to see that the family $\left(\sigma_{l}\right)_{l \in \mathbb{Z}_{4}} \cup\left(\tau_{l}\right)_{l \in \mathbb{Z}_{4}}$ is of type $\mathcal{D}_{4}^{(2)}$. We choose $g_{0}:=\mathrm{id}, g_{1}:=(69)(811)(1012), g_{2}:=$ (6 8)(10 12), $g_{3}:=\left(\begin{array}{ll}6 & 11\end{array}\right)\left(\begin{array}{l}8 \\ 9\end{array}\right)\left(\begin{array}{ll}10 & 12\end{array}\right)$ and $h_{l}:=\left(\begin{array}{ll}2 & 3\end{array}\right) g_{l}, l \in \mathbb{Z}_{4}$.

Let $v, w \in V-0$. We define $W:=\mathbb{C}$-span of $\left\{u_{l}, v_{l} \mid l \in \mathbb{Z}_{4}\right\}$, where $u_{l}, v_{l}$, are given by (14). The condition $q_{\sigma \sigma}=-1$, implies that $\rho\left(A_{2}\right)=\rho_{2}\left(A_{2}\right)=1$, because $\rho_{4}=\chi_{(i, i)} \otimes \operatorname{sgn}$ or $\chi_{(-i,-i)} \otimes$ sgn. This implies that $\rho_{2}=\chi_{\left(t_{2}, t_{2}\right)} \otimes \mu_{2}$, with $t_{2}=0$ or 1 , and $\mu_{2}=\epsilon$ or sgn. We consider two cases.

(a) $\rho_{2}=\chi_{\left(t_{2}, t_{2}\right)} \otimes \epsilon$. By straightforward computations, we can see that $W$ is a braided vector subspace of $M\left(\mathcal{O}_{\sigma}, \rho\right)$ of Cartan type with matrix of coefficients given by

$$
\left(\begin{array}{ll}
Q & Q \\
Q & Q
\end{array}\right), \text { where } Q=\left(\begin{array}{cccc}
-1 & -1 & -1 & 1 \\
-1 & -1 & -1 & 1 \\
1 & -1 & -1 & -1 \\
1 & -1 & -1 & -1
\end{array}\right)
$$

and Dynkin diagram given by (15), and $\operatorname{dim} \mathfrak{B}\left(\mathcal{O}_{\sigma}, \rho\right)=\infty$.

(b) $\rho_{2}=\chi_{\left(t_{2}, t_{2}\right)} \otimes$ sgn. We proceed in an analogous way.

Our next goal is to discard types with $n_{4}>0$ and non-trivial $\sigma_{o}$. The proof relies on the technique of the octahedral rack [AF2, Section 4].

Proposition 3.7. Let $\sigma \in \mathbb{S}_{m}$ of type $\left(1^{n_{1}}, 2^{n_{2}}, 4^{n_{4}}, \sigma_{o}\right)$, with $n_{4}>0$ and $\sigma_{o} \neq \mathrm{id}, \mathcal{O}$ the conjugacy class of $\sigma$ and $\rho \in \widehat{\mathbb{S}_{m}^{\sigma}}$. Then $\operatorname{dim} \mathfrak{B}(\mathcal{O}, \rho)=\infty$.

Proof. We assume that $q_{\sigma \sigma}=-1$, by Lemma 2.1. Hence $q_{e}=-1$ and $q_{o}=1$ - see Remark 3.2. Notice that $q_{e}=q_{A_{2}} q_{A_{4}}$. We consider two cases.

(I) Assume that $n_{4}=1$. Let $A_{1,4}=\left(\begin{array}{llll}j_{1} & j_{2} & j_{3} & j_{4}\end{array}\right)$ the 4-cycle appearing in the decomposition of $\sigma$ as product of disjoint cycles. We call $s_{1}=A_{1,4}$, 
$s_{2}=\left(\begin{array}{llll}j_{1} & j_{2} & j_{4} & j_{3}\end{array}\right), s_{3}=\left(\begin{array}{llll}j_{1} & j_{3} & j_{2} & j_{4}\end{array}\right), s_{4}=s_{2}^{-1}$ and $s_{5}=s_{3}^{-1}, s_{6}=s_{1}^{-1}$. Now, we define $\sigma_{l}:=A_{1} A_{2} s_{l} \sigma_{o}, \tau_{l}:=A_{1} A_{2} s_{l} \sigma_{o}^{-1} 1 \leq l \leq 6$. Then the family $\left(\sigma_{l}, \tau_{l}\right)_{1 \leq l \leq 6}$ is of type $\mathfrak{O}^{(2)}-$ see [AF2, Def. 4.7].

We choose $g:=\prod_{k \text { odd }} g_{A_{k}}-$ see (11). Thus, $g$ is an involution in $\mathbb{S}_{m}$ such that $g \sigma_{o} g=\sigma_{o}^{-1}$; then $g \sigma g=\tau_{1}$. Now, we compute

$$
\begin{aligned}
\rho\left(g^{-1} \sigma_{1} g\right) & =\rho\left(\tau_{1}\right)=\rho\left(A_{1} A_{2} s_{1} \sigma_{o}^{-1}\right)=\rho\left(A_{2} A_{4}\right) \rho\left(\sigma_{o}\right)^{-1}=q_{e} q_{o} \mathrm{Id}=-\mathrm{Id}, \\
\rho\left(\sigma_{6}\right) & =\rho\left(A_{1} A_{2} A_{4}^{-1} \sigma_{o}\right)=\rho\left(\left(A_{2} A_{4}\right)^{-1}\right) \rho\left(\sigma_{o}\right)=q_{e}^{-1} q_{o} \mathrm{Id}=-\mathrm{Id}, \\
\rho\left(g^{-1} \sigma_{6} g\right) & =\rho\left(A_{1} A_{2} A_{4}^{-1} \sigma_{o}^{-1}\right)=\rho\left(\left(A_{2} A_{4}\right)^{-1}\right) \rho\left(\sigma_{o}^{-1}\right)=q_{e}^{-1} q_{o}^{-1} \mathrm{Id}=-\mathrm{Id} .
\end{aligned}
$$

Then $\operatorname{dim} \mathfrak{B}(\mathcal{O}, \rho)=\infty$, by [AF2, Th. 4.11].

(II) Assume that $n_{4}=2$. Let $A_{1,4}=\left(\begin{array}{llll}j_{1} & j_{2} & j_{3} & j_{4}\end{array}\right)$ and $A_{2,4}=\left(\begin{array}{llll}j_{5} & j_{6} & j_{7} & j_{8}\end{array}\right)$ the two 4-cycles appearing in the decomposition of $\sigma$. Now, we proceed as in the previous case with $s_{1}=A_{1,4} A_{2,4}, s_{2}=\left(\begin{array}{llll}j_{1} & j_{2} & j_{4} & j_{3}\end{array}\right)\left(\begin{array}{llll}j_{5} & j_{6} & j_{8} & j_{7}\end{array}\right)$, $s_{3}=\left(\begin{array}{llll}j_{1} & j_{3} & j_{2} & j_{4}\end{array}\right)\left(\begin{array}{llll}j_{5} & j_{7} & j_{6} & j_{8}\end{array}\right), s_{4}=s_{2}^{-1}, s_{5}=s_{3}^{-1}$ and $s_{6}=s_{1}^{-1}$.

We finally discard most of the representations $\rho_{1}$ entering in $\rho$, see (5). We apply the technique of $\mathcal{D}_{3}$, see [AF2, Section 3], to the first proposition, and the the technique of the octahedron, see [AF2, Section 4], to the second proposition.

Proposition 3.8. Let $\sigma \in \mathbb{S}_{m}$ of type $\left(1^{n_{1}}, 2^{n_{2}}, 4^{n_{4}}, \sigma_{o}\right)$, with $n_{2}>0$, $\mathcal{O}$ the conjugacy class of $\sigma$ and $\rho \in \widehat{\mathbb{S}_{m}^{\sigma}}$. If $\operatorname{deg} \rho_{1}>1$, then $\operatorname{dim} \mathfrak{B}(\mathcal{O}, \rho)=\infty$.

Proof. We assume that $q_{\sigma \sigma}=-1$, by Lemma 2.1. Since $\operatorname{deg} \rho_{1}>1$ we have that $n_{1}>0$; actually $n_{1} \geq 3$. Let $A_{1,2}=\left(j_{1} j_{2}\right)$ be a transposition appearing in $\sigma$. There exists a $j_{3}$ such that $\sigma$ fixes $j_{3}$ because $n_{1}>0$. We define $\sigma_{1}:=\sigma, \sigma_{2}:=\left(\begin{array}{ll}j_{1} & j_{3}\end{array}\right)\left(j_{1} j_{2}\right) \sigma$ and $\sigma_{3}:=\left(\begin{array}{ll}j_{2} & j_{3}\end{array}\right)\left(\begin{array}{ll}j_{1} & j_{2}\end{array}\right) \sigma$. We choose $g_{1}=\mathrm{id}$, $g_{2}=\left(j_{2} j_{3}\right)$ and $g_{3}=\left(j_{1} j_{3}\right)$. Let $v, w$ be two linearly independent vectors in $V_{1}$, the vector space affording $\rho_{1}$. We define $W:=\operatorname{span}$ of $\left\{g_{l} v, g_{l} w \mid 1 \leq\right.$ $l \leq 3\}$. Then $W$ is a braided vector subspace of $M\left(\mathcal{O}_{\sigma}, \rho\right)$ isomorphic to $M\left(\mathcal{O}_{2}^{3}, \operatorname{sgn}\right) \oplus M\left(\mathcal{O}_{2}^{3}, \operatorname{sgn}\right)$, and $\operatorname{dim} \mathfrak{B}(W)=\infty-$ see [AHS, Th. 4.8] or [AF2, Th. 2.1]. Therefore, $\operatorname{dim} \mathfrak{B}(\mathcal{O}, \rho)=\infty$.

Proposition 3.9. Let $\sigma \in \mathbb{S}_{m}$ of type $\left(1^{n_{1}}, 2^{n_{2}}, 4^{n_{4}}, \sigma_{o}\right)$, with $n_{4}>0$, $\mathcal{O}$ the conjugacy class of $\sigma$ and $\rho \in \widehat{\mathbb{S}_{m}^{\sigma}}$. If $\operatorname{deg} \rho_{1}>1$, then $\operatorname{dim} \mathfrak{B}(\mathcal{O}, \rho)=\infty$.

Proof. We assume that $q_{\sigma \sigma}=-1$, by Lemma 2.1. Analogously to the previous result, we can construct a braided vector subspace of $M\left(\mathcal{O}_{\sigma}, \rho\right)$ isomorphic to $M\left(\mathcal{O}_{4}^{4}, \chi_{(-1)}\right) \oplus M\left(\mathcal{O}_{4}^{4}, \chi_{(-1)}\right)$. Then $\operatorname{dim} \mathfrak{B}(\mathcal{O}, \rho)=\infty$, by AHS, Th. 4.7]. 
Our final reduction is about the characters in cases (iii) and (iv) of the main Theorem. We apply the technique of the rack $\mathcal{D}_{3}^{(2)}$.

Proposition 3.10. Let $\sigma \in \mathbb{S}_{m}$ of type $\left(1^{n_{1}}, 2^{n_{2}}\right)$, with $n_{1}>0$, $\mathcal{O}$ the conjugacy class of $\sigma$ and $\rho \in \widehat{\mathbb{S}_{m}^{\sigma}}$. If

(i) $n_{2}=3$ and $\rho_{2}=\chi_{(3)} \otimes \epsilon$, or

(ii) $n_{2}=5$ and $\rho_{2}=\chi_{(5)} \otimes \epsilon$ or $\chi_{(5)} \otimes \operatorname{sgn}$,

then $\operatorname{dim} \mathfrak{B}(\mathcal{O}, \rho)=\infty$.

Proof. (i) We set $\sigma=(12)(34)(56)$ and define $\sigma_{0}:=\sigma, \sigma_{1}:=(12)(34)(57)$, $\sigma_{2}:=\sigma_{0} \triangleright \sigma_{1}, \tau_{0}:=(13)(24)(56), \tau_{1}:=\sigma_{2} \triangleright \tau_{0}$ and $\tau_{2}:=\sigma_{1} \triangleright \tau_{0}$. Then $\left(\sigma_{j}, \tau_{j}\right)_{j \in \mathbb{Z}_{3}}$ is a family of type $\mathcal{D}_{3}$ in $\mathcal{O}_{\sigma}$. We choose $g=(23)$. Thus, $\rho\left(g^{-1} \sigma g\right)=\rho\left(\tau_{0}\right)=\rho_{2}\left(A_{3,2} B_{1,2}\right) \mathrm{Id}=-1$, see Subsection 2.2. Therefore, $\operatorname{dim} \mathfrak{B}(\mathcal{O}, \rho)=\infty$, by [AF2, Th. 3.7].

(ii) We take $\sigma=(12)(34)(56)(78)(910)$ and define $\sigma_{0}:=\sigma, \sigma_{1}:=$ $(12)(34)(56)(78)(911), \sigma_{2}:=\sigma_{0} \triangleright \sigma_{1}$,

$$
\tau_{0}:=(13)(24)(57)(68)(910),
$$

$\tau_{1}:=\sigma_{2} \triangleright \tau_{0}$ and $\tau_{2}:=\sigma_{1} \triangleright \tau_{0}$. Then $\left(\sigma_{j}, \tau_{j}\right)_{j \in \mathbb{Z}_{3}}$ is a family of type $\mathcal{D}_{3}$ in $\mathcal{O}_{\sigma}$. We choose $g=(23)(67)$. Thus, $\rho\left(g^{-1} \sigma g\right)=\rho\left(\tau_{0}\right)=\rho_{2}\left(A_{5,2} B_{1,2} B_{3,2}\right) \operatorname{Id}=$ -1 , see Subsection 2.2. Hence, $\operatorname{dim} \mathfrak{B}(\mathcal{O}, \rho)=\infty$, by [AF2, Th. 3.7].

\section{REFERENCES}

[AF1] N. Andruskiewitsch and F. Fantino, On pointed Hopf algebras associated with unmixed conjugacy classes in $\mathbb{S}_{m}$, J. Math. Phys. 48 (2007), 033502-1 - 033502-26.

[AF2] - New techniques for pointed Hopf algebras, arXiv:0803.3486v1, 29 pp.

[AG] N. Andruskiewitsch and M. Graña, From racks to pointed Hopf algebras, Adv. Math. 178 (2003), $177-243$.

[AHS] N. Andruskiewitsch, I. Heckenberger and H.-J. Schneider, The Nichols algebra of a semisimple Yetter-Drinfeld module, arXiv:0803.2430v1.

[AS] N. Andruskiewitsch and H.-J. Schneider, Pointed Hopf Algebras, in "New directions in Hopf algebras", 1-68, Math. Sci. Res. Inst. Publ. 43, Cambridge Univ. Press, Cambridge, 2002.

[AZ] N. Andruskiewitsch and S. Zhang, On pointed Hopf algebras associated to some conjugacy classes in $\mathbb{S}_{n}$, Proc. Amer. Math. Soc. 135 (2007), 2723-2731.

[FK] S. Fomin and K. N. Kirillov, Quadratic algebras, Dunkl elements, and Schubert calculus, Progr. Math. 172, Birkhauser, (1999), pp. 146182.

[Gñ1] M. Graña, On Nichols algebras of low dimension, Contemp. Math. 267 (2000), $111-134$.

[Gñ2] Finite dimensional Nichols algebras of non-diagonal group type,zoo of examples available at http://mate.dm.uba.ar/ matiasg/zoo.html. 
[H] I. Heckenberger, Classification of arithmetic root systems, math.QA/0605795.

[HS] I. Heckenberger and H.-J. Schneider, Root systems and Weyl groupoids for semisimple Nichols algebras, arXiv:0807.0691.

[MS] A. Milinski and H-J. Schneider, Pointed Indecomposable Hopf Algebras over Coxeter Groups, Contemp. Math. 267 (2000), 215-236.

[S] Jean-Pierre Serre, Linear representations of finite groups, Springer-Verlag, New York 1977.

Facultad de Matemática, Astronomía y Física, Universidad Nacional de Córdoba, Ciem - COniCET, (5000) Ciudad Universitaria, Córdoba, Argentina

E-mail address: andrus@famaf .unc.edu.ar

E-mail address: fantino@famaf.unc.edu.ar

Department of Mathematics, Human University, Changsha 410082, P.R. CHINA

E-mail address: z9491@yahoo.com.cn 